\title{
Fast In-Plane Dynamics of a Beam with Unilateral Constraints
}

\author{
Anna Liakou'; Vincent Denoël, Aff.M.ASCE²; and Emmanuel Detournay, A.M.ASCE ${ }^{3}$
}

\begin{abstract}
A computationally efficient technique to simulate the dynamic response of a beam colliding with rigid obstacles is described in this paper. The proposed method merges three key concepts. First, a low-order discretization scheme that maximizes the number of nodes of the discrete model (where impacts are detected) at the expense of the degree of continuity of the constructed displacement field is used. Second, the constrained problem is transformed into an unconstrained one by formulating the impact by using a Signorini complementarity law involving the impulse generated by the collision and the preimpact and postimpact velocity linked through a coefficient of restitution. Third, Moreau's midpoint time-stepping scheme developed in the context of colliding rigid bodies is used to advance the solution. The algorithm is first validated on the nonimpact problem of a cantilever Rayleigh beam subjected to an impulsive discrete load. Then the problem of a cantilever beam vibrating between two (symmetrically located) stops is analyzed. Both cases of discrete and continuous obstacles are considered, and the numerical predictions are compared with published results or those obtained with a commercial code. DOI: 10.1061/ (ASCE)EM.1943-7889.0001175. (C) 2016 American Society of Civil Engineers.
\end{abstract}

\section{Introduction}

Mechanical systems colliding with rigid barriers are ubiquitous. Thus, there is significant interest in accurately predicting their response to impact. Although robust computational techniques now exist to simulate the constrained motion of rigid multibody systems (Brogliato 1996; Glocker 2001; Leine and Nijmeijer 2004; Acary 2008; Leine 2008), methods to analyze the impact behavior of continuous compliant systems remain an active area of research. Recognizing that beamlike structures are prevalent in industrial applications (Andrews et al. 1996; Bishop et al. 1996; Wagg and Bishop 2002; Ibrahim 2009), this paper focuses on formulating an efficient technique to compute the fast dynamics of a beam colliding with rigid obstacles.

Contact and impact problems are related, although essential differences exist in view of their differing static and dynamic natures. In unilateral contact problems, the constraints on the system are at the displacement level, with the contact forces being unknown a priori. In problems involving the impact of a structure with a rigid obstacle, the constraints are not only at the displacement level but also at the velocity level through the application of Newton's impact law, with the impulse generated by the impact being now unknown a priori.

As can be expected, numerous numerical techniques have been developed for simulating the constrained deflection of a beam (contact problem). They include finite-difference schemes using penalty methods for the contact forces (Klapper 1996; Goyal et al. 2008)

\footnotetext{
${ }^{1}$ Graduate Student, Dept. of Civil, Environmental, and Geo-Engineering, Univ. of Minnesota, 500 Pillsbury Dr. SE, Minneapolis, MN 55455.

${ }^{2}$ Associate Professor, Dept. of Architecture, Geology, Environment, and Constructions, Univ. of Liège, Chemin des Chevreuils, 1 Bât B 52/3, Liège 4000, Belgium.

${ }^{3}$ Professor, Dept. of Civil, Environmental, and Geo-Engineering, Univ. of Minnesota, 500 SE Pillsbury Dr. SE, Minneapolis, MN 55455 (corresponding author). E-mail: detou001@umn.edu

Note. This manuscript was submitted on June 1, 2016; approved on August 9, 2016; published online on October 31, 2016. Discussion period open until March 31, 2017; separate discussions must be submitted for individual papers. This paper is part of the Journal of Engineering Mechanics, (C) ASCE, ISSN 0733-9399.
}

and finite-element methods using different approaches for solving the contact problem (Simo 1985; Simo and Vu-Quoc 1986; Cardona and Geradin 1988; Belytschko et al. 2000).

Conversely, methods to simulate the impact of a beam with a rigid obstacle are still under development. One group of methods is based on the modal decomposition of the solution and on satisfaction of Newton's law at impact. In one approach, a velocity jump is imposed at the point of impact, which is restricted to coincide with a collocation point (Wagg 2004); in another, an impulse is applied wherever and whenever an impact occurs (Vyasarayani 2009; Vyasarayani et al. 2009, 2012). However, the predicted dynamic response is affected by the number of modes used to construct the solution, which should be adapted to the stiffness of the obstacle (Melcher et al. 2013). Hence, the impact of a beam with an infinitely rigid obstacle cannot be solved accurately from this standpoint.

A second group of methods is based on a variational formulation of the constrained beam problem (Paoli 2001; Pozzolini and Salaun 2011; Dumont and Paoli 2015). For space discretization, a Galerkin approximation (Dumont and Paoli 2015) or the method of lines (Dumont 2002) are used commonly. Different strategies are used to model the impact. One is to introduce a normal stiffness (Dumont 2002; Johansson 1997). Although appealing because of the simplicity of its implementation, this method introduces large reaction forces, because of the assumed large contact stiffness, that eventually cause difficulty in solving the contact problem (Baraff 1994; Dumont and Paoli 2015). Another strategy is to use a timestepping scheme with a predefined coefficient of restitution (Dumont 2003; Pozzolini and Salaun 2011; Dumont and Paoli 2015). However, the presence of the unilateral constraints and the necessity of minimizing numerical dissipation make the choice of the time-integration scheme a challenging task (Pozzolini and Salaun 2011). To cover all these requirements, multiple timeintegration schemes have been proposed, such as the backward differentiation formula (BDF), the Hilber-Hughes-Taylor (HHT- $\alpha$ ) method, and the implicit Newmark scheme [see Dumont (2002), Chen et al. (2012, 2013), Acary (2014), Bruls et al. (2014), and Depouhon et al. (2014) for a discussion on different time-stepping schemes].

This paper describes a fast and accurate method to simulate the impact of a Rayleigh beam against rigid obstacles that is also 


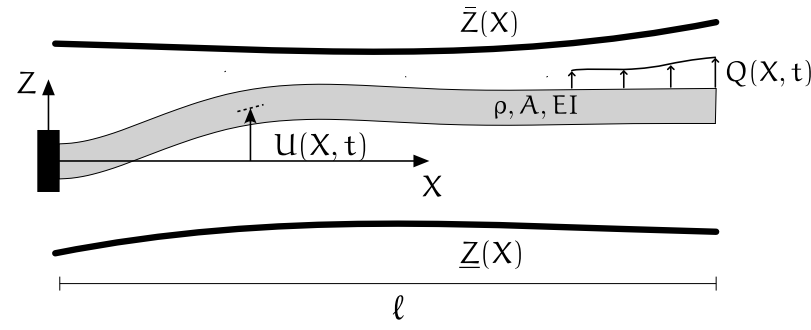

Fig. 1. Vibrating beam between two rigid walls

capable of efficiently managing multiple simultaneous contact points and prolonged contact times. The proposed method merges three key concepts. First, a low-order discretization scheme, equivalent to a representation of the beam as a Hencky (1920) chain, is used; such a scheme maximizes the number of nodes - the location of the points on the beam at which impact can be detected-at the expense of the degree of continuity of the constructed displacement field. Second, the constrained problem is transformed into an unconstrained one by formulating the impact by using a Signorini complementarity law involving the impulse generated by the collision and the preimpact and postimpact velocity linked through a coefficient of restitution. Third, Moreau's midpoint time-stepping scheme, a two-step algorithm developed in the context of colliding rigid bodies, is used to advance the solution. The combination of the Signorini law formulated at the velocity level and the Moreau midpoint scheme requires solving a nonlinear system of equations for the impulse and the preimpact and postimpact velocities whenever a node of the discrete beam is detected to be in contact (within a set tolerance) of an obstacle. The algorithm is validated first on the nonimpact problem of a cantilever Rayleigh beam subjected to an impulsive discrete load. Then the problem of a cantilever beam vibrating between two symmetrically located stops is analyzed. Both cases of discrete and continuous obstacles are considered, and the numerical predictions compared with published results or results obtained with a commercial code.

\section{Beam Subjected to Impact Loading}

\section{Problem Formulation}

Fig. 1 illustrates a beam vibrating between two rigid walls that restrict its motion. It is an example of the class of problems that one aims to simulate numerically. Consideration is limited in this study to beams with a doubly symmetric cross section that are subjected to transverse time-dependent loading acting in one of the planes of symmetry.

In Fig. 1, a Cartesian coordinate system $\{X, Y, Z\}$ with its origin at the left end of the beam is defined so that the horizontal $X$-axis corresponds to the centerline of the unstressed beam, and the $Y$ - and $Z$-axes to the principal axes of inertia. The $Z$-axis is vertical and pointing upward. In its usual one-dimensional representation, the beam of length $\ell$ is defined in the interval $0 \leq X \leq \ell$.

Let $U(X, t)$ denote the transverse displacement of the beam, a function of both position $X \in[0, \ell]$ and time $t>0$. It is governed by the classic equation of motion (Love 2013; Rao 2007; Han et al. 1999), as follows:

$$
\rho A \frac{\partial^{2} U}{\partial t^{2}}-\rho I \frac{\partial^{4} U}{\partial X^{2} \partial t^{2}}+E I \frac{\partial^{4} U}{\partial X^{4}}=Q+\Phi
$$

where $E=$ elastic modulus; $I=$ moment of inertia; $A=$ crosssectional area; and $\rho=$ density. For simplicity, all properties have been assumed to be uniform. Two kinds of loading are considered: a known body-type force $Q(X, t)$, where $Q$ is defined per unit length of the beam; and a priori unknown impact forces $\Phi(X, t)$ resulting from collisions of the beam with the upper and lower obstacles. Both $Q(X, t)$ and $\Phi(X, t)$ are transverse forces acting in the plane $(X, Z)$. The quantities $U, Q$, and $\Phi$ have the same sign convention, consistent with $Z$. The governing equation is supplied with four boundary conditions (prescribing either the transverse displacement or shear force, and either the inclination or the bending moment at both ends) and two initial conditions [e.g., $U(X, 0)=\partial U(t, 0) / \partial t=0]$.

The presence of the two rigid walls constrains the motion of the beam. Let $\bar{Z}(X)$ and $\underline{z}(X)$ denote the signed clearance between the beam in its initial configuration and the upper and lower obstacles, respectively. Hence, the transverse displacement of the beam is constrained to the interval

$$
\underline{z}(X) \leq U(X, t) \leq \bar{Z}(X), \quad 0<X<\ell, t>0
$$

The impact law, required to close the formulation of the problem, is described subsequently, together with some restrictions on the nature of the collision between the beam and the walls.

\section{Scaling}

By introducing characteristic quantities (namely, a length $\ell_{*}$, a time $t_{*}$, a displacement $U_{*}$, a body force $Q_{*}$, and an impulse $\Lambda_{*}$ ), such as

$$
\ell_{*}=\ell, \quad t_{*}=\sqrt{\frac{\rho A \ell^{4}}{E I}}, \quad U_{*}=\frac{\ell^{4} Q_{*}}{E I}, \quad \Lambda_{*}=\ell t_{*} Q_{*}
$$

governing Eq. (1) can be scaled according to

$$
\ddot{u}-\beta^{2} \frac{\partial^{2} \ddot{u}}{\partial x^{2}}+\frac{\partial^{4} u}{\partial x^{4}}=q+\phi
$$

with the various dimensionless quantities and parameters appearing in Eq. (4) defined as

$$
\begin{aligned}
x & =\frac{X}{\ell}, \quad \tau=\frac{t}{t_{*}}, \quad u=\frac{U}{U_{*}}, \quad q=\frac{Q}{Q_{*}}, \quad \phi=\frac{\Phi}{Q_{*}}, \\
\beta^{2} & =\frac{I}{A \ell^{2}}
\end{aligned}
$$

The standard notation $\dot{u}=\partial u / \partial \tau$ has been adopted, and $u^{\prime}=$ $\partial u / \partial x$ also will be used at some point. The free scale $Q_{*}$ can, in principle, be chosen arbitrarily but should be selected so that $\|q\|$ is of order $O(1)$. This is not possible, however, if the imposed loading is of an impulsive nature, e.g., $Q(X, t)=\Lambda \delta\left(X-X_{o}\right) \delta(t)$, where $\delta$ is the Dirac $\delta$-function, and $X_{o} \in[0, \ell]$. In this case, the characteristic impulse $\Lambda_{*}=\Lambda$, so that $Q_{*}$ is chosen as $Q_{*}=\Lambda / \ell t_{*}$, and $U_{*}=\ell^{3} \Lambda_{*} / E I t_{*}$. In any case, $\Lambda_{*}$ is used to scale the impulsive forces induced by collision of the beam with the surrounding walls. Eq. (5) describes the dynamic response of a Rayleigh beam, but it degenerates to the governing equation of a Euler-Bernoulli beam when $\beta$ tends to 0 .

It is now assumed that the forces generated by the collision of the beam with the rigid walls $\underline{z}(x)$ and $\bar{z}(x)$ (where $\underline{z}=\underline{z} / U_{*}$, and $\left.\bar{z}=\bar{Z} / U_{*}\right)$ can be viewed as discrete and impulsive. In other words, it is assumed not only that the length of contact at impact is very small compared with the beam length $\ell$ but also that the duration of contact is negligible compared with $t_{*}$, a measure of the period of the first vibration mode of the beam. These assumptions not only limit the impact velocity of the beam, which should 
be small enough to satisfy the imposed restrictions, but also constrain the geometry of the rigid wall. Under these restrictive conditions, the impact forces $\phi(x, \tau)$ can be expressed as

$$
\phi(x, \tau)=\sum_{l=1}^{m(\tau)} \lambda_{l} \delta\left(x-x_{l}\right) \delta\left(\tau-\tau_{l}\right)
$$

where $\lambda_{l}=$ defined by scaling the original impulses with $\Lambda_{*}$. Impulses $\lambda_{l}$, locations $x_{l}$, and times $\tau_{l}$ of the impacts are unknown a priori. They are calculated as part of the solution by accounting for the constraint

$$
\underline{z}(x) \leq u(x, \tau) \leq \bar{z}(x), \quad 0<x<1, \quad \tau>0
$$

and Newton's coefficient of restitution $e$

$$
\begin{aligned}
\dot{u}\left(x_{l}, \tau_{l}^{+}\right) & =-e \dot{u}\left(x_{l}, \tau_{l}^{-}\right) \quad \text { with } \quad \underline{z}\left(x_{l}\right)=u\left(x_{l}, \tau_{l}\right) \quad \text { or } \\
\bar{z}\left(x_{l}\right) & =u\left(x_{l}, \tau_{l}\right)
\end{aligned}
$$

where it has been noted again that the contact condition $\underline{z}=u$ or $\bar{z}=u$ is restricted to be discrete in time and space. Eqs. (4), (7), and (8), together with initial conditions at $\tau=0$, boundary conditions at $x=0$ and $x=1$, and known loading $q(x, \tau)$ for $x \in[0,1]$ and $\tau>0$, form a closed system of equations for the field $u(x, \tau)$. The challenge in solving numerically this system of equations is the robust and accurate determination of the impact parameters $\lambda_{l}$ and $\tau_{l}$, as the location of the collision events will be restricted to coincide with the nodes of the discretization mesh.

\section{Semidiscretized Equations of Motion}

When devising a spatial discretization scheme of the equation of motion Eq. (1), there is a trade-off between the number of nodes at which $u$ is calculated and the order of the polynomial that approximates the displacement field between the nodes. In this study, motivated by the desire to capture the location of the impacts accurately and recognizing that an algorithm that limits the collision detection to the nodes of the mesh is expected to be computationally efficient, one favors a discretization scheme with a low-order interpolation function but with many nodes. The Hencky chain model of a beam, which consists of rigid segments connected by elastic hinges (Hencky 1920; Salvadori 1951; Challamel et al. 2015; Wang et al. 2015) is such a scheme, one that maximizes the number of nodes. However, starting from a different representation based on a dual-discretization scheme, one obtains discretized equations of motion identical to those governing the response of a Hencky chain.

\section{Dual Discretization of a Rayleigh Beam}

Inspired by Spillmann and Teschner (2007), the authors use a low-order dual-discretization scheme to formulate the approximated equations of motion of a Rayleigh beam. The centerline of the beam is discretized by a chain of $n+1$ equally spaced nodes located at $x_{i}=(i-1) \Delta x, i \in[1, n+1]$, where $\Delta x=1 / n$ denotes the distance between two adjacent nodes. Let $u_{i}(\tau)$ denote the transverse displacement, and $q_{i}(\tau)$ the force per unit length at node $i$.

A set of $n$ auxiliary midnodes $i+1 / 2, i=1, n$ located at middistance between nodes $i$ and $i+1$ also is introduced. The inclination $\theta_{i+1 / 2}, i=1, n$ of the centerline is assigned to these midnodes; $\theta_{i+1 / 2}$ is related simply to the nodal displacements $u_{i}$ and $u_{i+1}$ according to

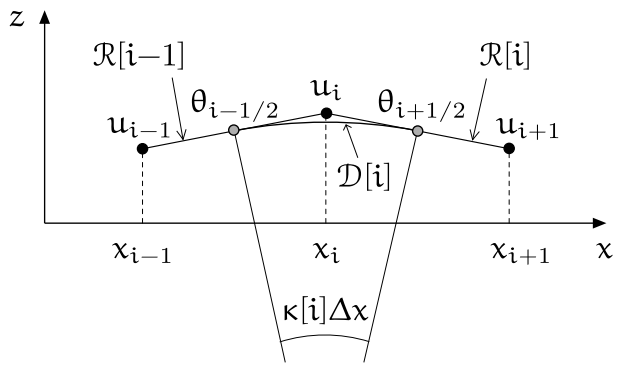

Fig. 2. Dual discretization of a beam

$$
\theta_{i+(1 / 2)}=\frac{u_{i+1}-u_{i}}{\Delta x}
$$

In Fig. 2, two types of elements are introduced: rigid $\mathcal{R}$-elements characterized by a rigid body motion (in the approximation of beam theory); and deformable $\mathcal{D}$-elements characterized by bending and by a nonuniform rotation of the center line. The discretized beam is thus seen as the superposition of these two types of elements, which are staggered as shown in Fig. 2. There are $n \mathcal{R}$-elements and $n+1 \mathcal{D}$-elements. Element $\mathcal{R}[i]$ is defined by the two end nodes $i$ and $i+1$, whereas element $\mathcal{D}[i]$, for $i=2, \ldots, n$, is delimited by the two midnodes $i-1 / 2$ and $i+1 / 2$. Elements $\mathcal{D}[1]$ and $\mathcal{D}[n+1]$ are half-elements defined by nodes 1 and $3 / 2$ and by nodes $n+1 / 2$ and $n+1$, respectively.

The $\mathcal{R}$-elements are characterized by a linear variation of the transverse displacement $u$ and of the force density $q$ between their two end nodes at any time $\tau$, and $\mathcal{D}$-elements by a linear variation of $\theta$. Thus, a $\mathcal{D}$-element has a uniform curvature. It is convenient to define the following vectors as

$$
\begin{array}{ll}
\mathbf{u}^{T}[i]=\left\{u_{i}, u_{i+1}\right\}, & \boldsymbol{\theta}^{T}[i]=\left\{\theta_{i-12}, \theta_{i+12}\right\}, \\
\mathbf{q}^{T}[i]=\left\{q_{i}, q_{i+1}\right\}, & \mathbf{N}^{T}(\xi)=\{1-\xi, \xi\}
\end{array}
$$

noting that $\mathbf{u}[i]$ and $\mathbf{q}[i]$ are assigned to element $\mathcal{R}[i]$, and $\boldsymbol{\theta}[i]$ to element $\mathcal{D}[i]$. Hence, displacement $u(\xi, \tau)$ along element $\mathcal{R}[i]$, $i=1, n$, can be expressed as

$$
u(\xi, \tau)=\mathbf{N}^{T}(\xi) \cdot \mathbf{u}[i] \quad \xi=\frac{x-x_{i}}{\Delta x}, \quad \xi \in[0,1]
$$

whereas inclination $\theta(\xi, \tau)$ along element $\mathcal{D}[i]$ centered at node $i=2, n$ reads

$$
\theta(\xi, \tau)=\mathbf{N}^{T}(\xi) \cdot \boldsymbol{\theta}[i] \quad \xi=\frac{x-x_{i-1 / 2}}{\Delta x}, \quad \xi \in[0,1]
$$

with curvature $\kappa[i]$ given by

$$
\kappa[i]=\frac{\theta_{i+1 / 2}-\theta_{i-1 / 2}}{\Delta x}=\frac{u_{i+1}-2 u_{i}+u_{i-1}}{\Delta x^{2}}
$$

The expression for the curvature of elements $\mathcal{D}[1]$ and $\mathcal{D}[n]$ depends on the boundary conditions. As illustrated in Fig. 2, the $\mathcal{R}$-elements correspond to chords, and the staggered $\mathcal{D}$-elements to osculating arcs of circle.

The specificity of this discretization scheme consists, therefore, in using the same interpolation functions for both the displacement and the rotation fields. This is an essential difference with classical numerical methods, such as finite elements, in which the order of interpolation of the differentiated fields (rotation) is by nature smaller than that of the fundamental ones (displacements). The current scheme might be seen as a low-order interpolation of both 
the displacement and rotation fields, in the manner of a state-space formulation, without a strong enforcement of the kinematic relation $\theta=\partial u / \partial x$. A second difference with classical methods consists in the offset between the two grids used for the two unknown fields.

\section{Kinetic and Potential Energy}

Kinetic energy $\mathcal{T}$ consists of contributions from both the rigid and the deformable elements, i.e.

$$
\mathcal{T}(\tau)=\sum_{i=1}^{n} T_{\mathrm{R}}[i]+\sum_{i=1}^{n+1} T_{\mathcal{D}}[i]
$$

The kinetic energy of elements $\mathcal{R}[i]$ and $\mathcal{D}[i]$ is given, respectively, by

$T_{\mathrm{R}}[i]=\frac{1}{2} \Delta x \dot{\mathbf{u}}[i]^{T} \cdot \mathbf{A} \dot{\mathbf{u}}[i], \quad T_{\mathcal{D}}[i]=\frac{1}{2} \Delta x \beta^{2} \dot{\boldsymbol{\theta}}[i]^{T} \cdot \mathbf{A} \dot{\boldsymbol{\theta}}[i]$

with

$$
\mathbf{A}=\int_{0}^{1} \mathbf{N}^{T} \mathbf{N} d \xi=\frac{1}{6}\left[\begin{array}{ll}
2 & 1 \\
1 & 2
\end{array}\right]
$$

Hence,

$$
\begin{gathered}
T_{\mathrm{R}}[i]=\frac{1}{6 n}\left(\dot{u}_{i}^{2}+\dot{u}_{i} \dot{u}_{i+1}+\dot{u}_{i+1}^{2}\right), \quad i=1, n \\
T_{\mathcal{D}}[i]=\frac{n \beta^{2}}{6}\left(\dot{u}_{i-1}^{2}+\dot{u}_{i}^{2}+\dot{u}_{i+1}^{2}-\dot{u}_{i-1} \dot{u}_{i}-\dot{u}_{i} \dot{u}_{i+1}-\dot{u}_{i-1} \dot{u}_{i+1}\right), \\
i=2, n
\end{gathered}
$$

The kinetic energy of the two end elements $\mathcal{D}[1]$ and $\mathcal{D}[n+1]$ depends on the boundary conditions at the extremities of the beam. For example, if $u=u^{\prime}=0$ at $x=0$, then $T_{\mathcal{D}}[1]=n \beta^{2} \dot{u}_{2}^{2} / 6$.

The potential energy consists of contributions from internal (moment) and external forces (distributed forces $q$ ) acting on the beam, i.e.

$$
\mathcal{V}=\sum_{i=1}^{n} V_{\mathrm{E}}[i]+\sum_{i=1}^{n+1} V_{\mathrm{I}}[i]
$$

with

$$
\begin{gathered}
V_{\mathrm{E}}[i]=-\Delta x \mathbf{q}[i]^{T} \cdot \mathbf{A} \mathbf{u}[i], \quad i=1, n \\
V_{\mathrm{I}}[i]=\frac{1}{2} \Delta x \kappa^{2}[i], \quad i=2, n
\end{gathered}
$$

Hence,

$V_{\mathrm{E}}[i]=-\frac{1}{6 n}\left[\left(2 q_{i}+q_{i+1}\right) u_{i}+\left(q_{i}+2 q_{i+1}\right) u_{i+1}\right], \quad i=1, n$

$$
V_{\mathrm{I}}[i]=\frac{n^{3}}{2}\left(u_{i-1}-2 u_{i}+u_{i+1}\right)^{2}, \quad i=2, n
$$

Again, the internal energy of $\mathcal{D}[1]$ and $\mathcal{D}[n+1]$ depends on the boundary conditions at the extremities of the beam; if $u=u^{\prime}=0$ at $x=0$, then $V_{\mathrm{I}}[1]=n^{3} u_{2}^{2} / 2$.

\section{Discrete Equations of Motion}

The Lagrangian for the discrete model is thus of the form $\mathscr{L}(\mathbf{u}, \dot{\mathbf{u}})=\mathcal{T}(\dot{\mathbf{u}})-\mathcal{V}(\mathbf{u})$, and the corresponding Euler-Lagrange equations are

$$
\frac{d}{d \tau} \frac{\partial \mathcal{T}}{\partial \dot{\mathbf{u}}}+\frac{\partial \mathcal{V}}{\partial \mathbf{u}}=\boldsymbol{\phi}
$$

where $\phi=$ set of impulsive forces acting at the nodes of the discrete model, resulting from impact with the rigid obstacles. In view of Eqs. (14) and (18)

$\frac{d}{d \tau} \frac{\partial}{\partial \dot{\mathbf{u}}}\left[\sum_{i=1}^{n} T_{\mathrm{R}}[i]+\sum_{i=1}^{n+1} T_{\mathcal{D}}[i]\right]+\frac{\partial}{\partial \mathbf{u}}\left[\sum_{i=1}^{n} V_{\mathrm{E}}[i]+\sum_{i=1}^{n+1} V_{\mathrm{I}}[i]\right]=\boldsymbol{\phi}$

The explicit expressions for the various terms entering in the discrete Euler-Lagrange Eq. (25) are

$$
\begin{array}{rlr}
\frac{d}{d \tau}\left(\frac{\partial T_{\mathrm{R}}[i]}{\partial \dot{\mathbf{u}}}\right) & =\mathbf{M}_{\mathrm{R}}[i]\left\{\begin{array}{c}
\ddot{u}_{i} \\
\ddot{u}_{i+1}
\end{array}\right\} \quad \text { with } \\
\mathbf{M}_{\mathrm{R}}[i] & =\frac{1}{6 n}\left[\begin{array}{cc}
2 & 1 \\
1 & 2
\end{array}\right], \quad i=1, n
\end{array}
$$

$$
\begin{aligned}
& \frac{d}{d \tau}\left(\frac{\partial T_{\mathcal{D}}[i]}{\partial \dot{\mathbf{u}}}\right)=\mathbf{M}_{\mathcal{D}}[i]\left\{\begin{array}{c}
\ddot{u}_{i-1} \\
\ddot{u}_{i} \\
\ddot{u}_{i+1}
\end{array}\right\} \text { with } \\
& \mathbf{M}_{\mathcal{D}}[i]=\frac{n \beta^{2}}{6}\left[\begin{array}{ccc}
2 & -1 & -1 \\
-1 & 2 & -1 \\
-1 & -1 & 2
\end{array}\right], \quad i=2, n \\
& \frac{\partial V_{\mathrm{I}}[i]}{\partial \mathbf{u}}=\mathbf{K}_{\mathcal{D}}[i]\left\{\begin{array}{c}
u_{i-1} \\
u_{i} \\
u_{i+1}
\end{array}\right\} \text { with } \\
& \mathbf{K}_{\mathcal{D}}[i]=n^{3}\left[\begin{array}{ccc}
1 & -2 & 1 \\
-2 & 4 & -2 \\
1 & -2 & 1
\end{array}\right], \quad i=2, n
\end{aligned}
$$

$$
\begin{aligned}
\frac{\partial V_{\mathrm{E}}[i]}{\partial \mathbf{u}} & =-\mathbf{L}_{\mathrm{R}}[i]\left\{\begin{array}{c}
q_{i} \\
q_{i+1}
\end{array}\right\} \quad \text { with } \quad \mathbf{L}_{\mathrm{R}}[i]=\frac{1}{6 n}\left[\begin{array}{ll}
2 & 1 \\
1 & 2
\end{array}\right], \\
i & =1, n
\end{aligned}
$$

As discussed previously, the expressions for $\mathbf{M}_{\mathcal{D}}[i]$ and $\mathbf{K}_{\mathcal{D}}[i]$ when $i=1$ or $i=n+1$ depend on the nature of the boundary conditions at $x=0$ and $x=1$, respectively. Finally, the various terms are assembled to yield the semidiscretized equation of motion

$$
\mathbf{M u ̈}+\mathbf{K u}=\mathbf{q}+\boldsymbol{\phi}
$$

where $\mathbf{q}=$ nodal forces resulting from the assembly of $\mathbf{L}_{\mathcal{R}}[i] \mathbf{q}[i]$; and $\boldsymbol{\phi}=$ impulsive nodal forces associated with impact. The preceding equations reduce to the equations of a Euler-Bernoulli beam when $\beta^{2} \rightarrow 0$. In the static limit with no impact, the discretized Eq. (30) is actually identical to those derived on the basis of a Hencky chain representation of the beam (Salvadori 1951; Challamel et al. 2015; Wang et al. 2015). 


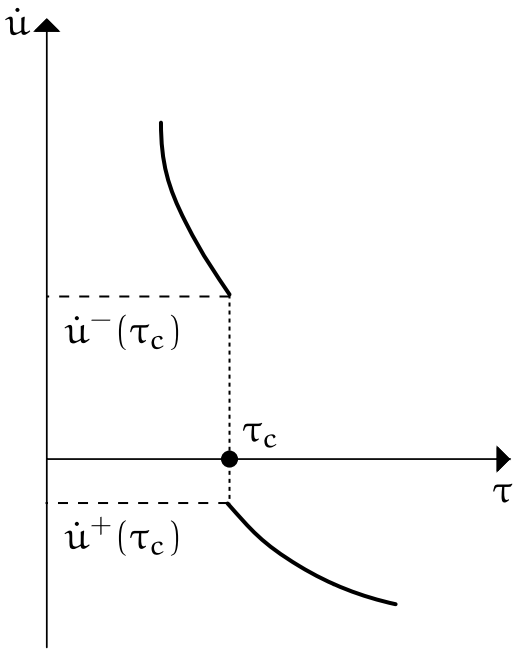

(a)

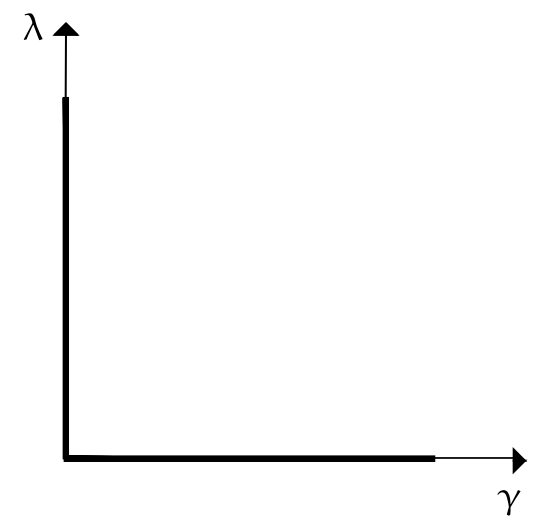

(b)

Fig. 3. (a) Velocity of bounded variations; (b) Newton impact law formulated as a Signorini complementarity law

\section{Impact Management}

The existence of a contact and/or an impact is described by unilateral (or inequality) constraints. Special treatment of the problem is required in this case because of the nonsmooth conditions. A numerical scheme based on Moreau time-stepping scheme is suggested.

\section{Decomposition of Motion and Forces in Smooth and Nonsmooth Parts}

To handle velocity discontinuities caused by the impulsive forces generated by collisions of the beam with obstacles, the system of Eq. (30) is transformed into measured differential equations (Glocker 2001; Leine and Nijmeijer 2004)

$$
\mathbf{M} d \dot{\mathbf{u}}+\mathbf{K} \mathbf{u} d t=\mathbf{q}(t) d t+d \mathbf{p}
$$

where $d \dot{\mathbf{u}}$ and $d \mathbf{p}=$ bounded differential measures of velocity and momentum, respectively. The differential measure $d \mathbf{p}$ of the discrete momentum can be expressed as

$$
d \mathbf{p}=\lambda d \eta
$$

where $\lambda=$ impulse arising from impacts during the time interval $d t$; and $d \eta=$ atomic differential measure [e.g., $d \eta=\delta\left(t_{c}\right) d t$ if only one such impact takes place during $d t$ at time $t_{c}$ ]. The differential measure of velocity $d \dot{\mathbf{u}}$ can be expressed as

$$
d \dot{\mathbf{u}}=\ddot{\mathbf{u}} d t+\left(\dot{\mathbf{u}}^{+}-\dot{\mathbf{u}}^{-}\right) d \eta
$$

where $\ddot{\mathbf{u}}=$ acceleration of the smooth background motion; and $\dot{\mathbf{u}}^{+}-\dot{\mathbf{u}}^{-}=$velocity jump associated with the impulse $\boldsymbol{\lambda}$.

By using these decompositions of momentum and velocity and considering that the external force $q(t)$ is continuous in time, the equation of motion [Eq. (31)] can be decomposed in terms of a smooth and a nonsmooth part (Leine and Nijmeijer 2004)

$$
\begin{gathered}
\mathbf{M} \ddot{\mathbf{u}}+\mathbf{K u}=\mathbf{q} \\
\mathbf{M}\left(\mathbf{u}^{+}-\mathbf{u}^{-}\right)=\boldsymbol{\lambda}
\end{gathered}
$$

where Eq. (34) describes the balance of forces for nonimpulsive motion, and Eq. (35) is the impact equation of motion.

\section{Newton Impact Law}

Consider a collision between the beam and an obstacle taking place at position $\xi=\xi_{i}$ at time $\tau=\tau_{c}$. According to Newton's impact law, the velocity jump $\dot{u}_{i}^{+}\left(\tau_{c}\right)-\dot{u}_{i}^{-}\left(\tau_{c}\right)=-(1+e) \dot{u}_{i}^{-}\left(\tau_{c}\right)$, where $e$ is the coefficient of restitution. This law, in combination with the equations of motion, determines the magnitude of the impulse $\lambda_{i}\left(\tau_{c}\right)$ generated at impact point $\xi_{i}$.

Two gap vectors, $\mathbf{g}=\mathbf{u}-\underline{\mathbf{z}}$ and $\overline{\mathbf{g}}=\overline{\mathbf{z}}-\mathbf{u}$, are introduced to measure the relative distance between the beam and the lower and upper obstacles, respectively: $g_{i} \geq 0$ and $\bar{g}_{i} \geq 0, i=1, n+1$. Evidently, $\dot{\mathbf{g}}=\dot{\mathbf{u}}$, and $\dot{\overline{\mathbf{g}}}=-\dot{\mathbf{u}}$. The collision event at $\xi=\xi_{i}$ and $\tau=\tau_{c}$ corresponds, therefore, to either $\underline{g}_{i}\left(\tau_{c}\right)=0$ or $\bar{g}_{i}\left(\tau_{c}\right)=0$. Attention is focused on collisions of the beam with the lower obstacle. Newton's impact law, now rewritten in terms of the gap [i.e., $\underline{g}_{i}^{+}\left(\tau_{c}\right)=-e \dot{g}_{i}^{-}\left(\tau_{c}\right)$ ] and the associated impulse $\lambda_{i}\left(\tau_{c}\right)$ can be formulated as a Signorini law

$$
\begin{aligned}
& \underline{\gamma}_{i}\left(\tau_{c}\right) \geq 0, \quad \lambda_{i}\left(\tau_{c}\right) \geq 0, \quad \gamma_{i}\left(\tau_{c}\right) \lambda_{i}\left(\tau_{c}\right)=0, \quad \text { with } \\
& \quad \underline{g}_{i}\left(\tau_{c}\right)=0
\end{aligned}
$$

where

$$
\underline{\gamma}_{i}\left(\tau_{c}\right)=\dot{g}_{i}^{+}\left(\tau_{c}\right)+e \underline{\dot{g}}_{i}^{-}\left(\tau_{c}\right)
$$

The preceding complementarity condition is interpreted as follows. Under the condition that the contact is closed at $\xi=\xi_{i}$ and at $\tau=\tau_{c}\left[g_{i}\left(\tau_{c}\right)=0\right]$, an impulse $\lambda_{i}\left(\tau_{c}\right)>0$ induces a jump in the relative velocity $\underline{g}_{i}\left(\tau_{c}\right)$, i.e., $\underline{g}_{i}^{+}\left(\tau_{c}\right)=-e \underline{\dot{g}}_{i}^{-}\left(\tau_{c}\right)$, evidently implying that $\dot{u}_{i}^{+}\left(\tau_{c}\right)=-e \dot{u}_{i}^{-}\left(\tau_{c}\right)$. Conversely, $\lambda_{i}=0$ implies that it is a grazing impact; thus, Newton's impact law needs to be relaxed, and $\dot{u}_{i}^{+}\left(\tau_{c}\right)>-e \dot{u}_{i}^{-}\left(\tau_{c}\right)$. The usual representation of Signorini's law, as applied to the impact problem, is sketched in Fig. 3(b); the possible states $\left\{\gamma_{i}, \lambda_{i}\right\}$ characterizing an impact are represented by the two thick lines on the positive parts of the two axes.

By using proximal point formalism, the complementarity condition Eq. (36) can be replaced advantageously by a projective equation. Noting that the proximal point of a convex $\operatorname{set} \mathcal{C}$ to a point $\mathbf{z}$ is the point $\mathbf{x}$ of $\mathcal{C}$ that is closest to $\mathbf{z}$ [i.e., if $\mathbf{z}$ is outside $\mathcal{C}$, the proximal point is on the boundary of $\mathcal{C}$ that is closest to $\mathbf{z}$; but if 
$\left.\mathbf{z} \in \mathcal{C}, \mathbf{z}=\operatorname{prox}_{\mathcal{C}}(\mathbf{z})\right]$, the proximal point function for the particular case $\mathcal{C}=\mathbb{R}^{+}$is simply

$$
\operatorname{prox}_{\mathbb{R}^{+}}(\zeta)= \begin{cases}\zeta & \text { if } \zeta>0 \\ 0 & \text { if } \zeta \leq 0\end{cases}
$$

In other words, $\operatorname{prox}_{\mathbb{R}^{+}}(\zeta)=\max (\zeta, 0)$. Signorini's law Eq. (37) can be rewritten as

$$
\lambda_{i}=\operatorname{prox}_{\mathbb{R}^{+}}\left(\lambda_{i}-r \underline{\gamma}_{i}\right)
$$

where $r>0=$ penalty parameter. This projective equation satisfies all the requirements of the impact law when the contact is closed, i.e., $g_{i}\left(\tau_{c}\right)=0$. Indeed, $\lambda_{i}>0$ implies that Eq. (39) reduces to $\lambda_{i}=\operatorname{prox}_{\mathbb{R}^{+}}\left(\lambda_{i}\right)$, indicating that $\gamma_{i}=0$. Conversely, $\lambda_{i}=0$ entails that $\operatorname{prox}_{\mathbb{R}^{+}}\left(\lambda_{i}-r \gamma_{i}\right)=0$ and thus that $\gamma_{i}>0$, as $r>0$.

\section{Time-Stepping Algorithm}

Integration of the momentum balance Eq. (31) over a finite time interval $\left\{\tau_{k}, \tau_{k+1}\right\}$ yields

$\dot{\mathbf{u}}_{k+1}-\dot{\mathbf{u}}_{k}=-\mathbf{M}^{-1} \mathbf{K} \int_{\tau_{k}}^{\tau_{k+1}} \mathbf{u} d t+\mathbf{M}^{-1}\left(\int_{\tau_{k}}^{\tau_{k+1}} \mathbf{q} d t+\boldsymbol{\lambda}\right)$

where $\dot{\mathbf{u}}_{k}$ and $\dot{\mathbf{u}}_{k+1}=$ nodal velocity at discrete times $\tau_{k}$ and $\tau_{k+1}$, respectively; and $\lambda$ contains the nodal impulses at each degreeof-freedom resulting from collision of the beam with the obstacles during time step $\Delta \tau=\tau_{k+1}-\tau_{k}$. The time-stepping algorithm is based on the Moreau midpoint scheme (Moreau and Panagiotopoulos 1988), one of the classic algorithms used to solve the motion equations for rigid body mechanics with impact. This scheme has several features. First, it operates at the level of velocity and momentum, not at the level of accelerations and forces that become infinite at impact. Second, whenever a collision is detected during a time step, the impulse of the impact force and the velocity discontinuity are calculated iteratively during that time step.

Moreau's algorithm is an Index 2 time-stepping scheme. Given displacement $\mathbf{u}_{k}$ and velocity $\dot{\mathbf{u}}_{k}$ at time $\tau_{k}$, the calculation of $\mathbf{u}_{k+1}$ and $\dot{\mathbf{u}}_{k+1}$ at time $\tau_{k+1}=\tau_{k}+\Delta \tau$ proceeds in two steps. In the first step, the displacement $\mathbf{u}_{k+1 / 2}$ at midpoint time $\tau_{k+1 / 2}=$ $\left(\tau_{k}+\tau_{k+1}\right) / 2$ is calculated by using the explicit scheme

$$
\mathbf{u}_{k+1 / 2}=\mathbf{u}_{k}+\frac{1}{2} \dot{\mathbf{u}}_{k} \Delta \tau
$$

and the gap distance vectors are updated accordingly, thus leading to $\underline{\mathbf{g}}_{k+1 / 2}$ and $\overline{\mathbf{g}}_{k+1 / 2}$. The midpoint state is then used to determine the contact set $\underline{\mathcal{I}}_{k+1 / 2}=\left\{i \mid \underline{\mathbf{g}}_{k+1 / 2} \leq \varepsilon\right\}$, which identifies the nodes of the beam where an impact is detected within tolerance $\varepsilon$. For simplicity of presentation, it is assumed that all the impacts during this time interval $\Delta t$ involve collision of the beam with the lower obstacle; the development can be generalized readily for the case of unilateral constraints on both sides of the beam. In the second step, velocity $\dot{\mathbf{u}}_{k+1}$ and impulse $\boldsymbol{\lambda}_{k+1}$ are computed by using the momentum balance in Eq. (40)

$$
\dot{\mathbf{u}}_{k+1}-\dot{\mathbf{u}}_{k}=\mathbf{M}^{-1}\left[-\left(\mathbf{K} \mathbf{u}_{k+1 / 2}-\mathbf{q}_{k+1 / 2}\right) \Delta \tau+\boldsymbol{\lambda}_{k+1}\right]
$$

where the integrals in Eq. (40) have been evaluated by using the trapezoidal rule, and Newton's impact law is now formulated by using the projective equation

$$
\lambda_{k+1}=\operatorname{prox}_{\mathbb{R}^{+}}\left[\boldsymbol{\lambda}_{k+1}-r \underline{\boldsymbol{\gamma}}_{k+1}\right]
$$

where $\underline{\boldsymbol{\gamma}}_{i, k+1}=\dot{u}_{i, k+1}+e \dot{u}_{i, k}$ with $i \in \underline{I}_{k+1 / 2}$; and $r \sim \max \left(\mathbf{M}_{i i}^{-1}\right)=$ penalty parameter. In view of the nonlinear nature of Eq. (43), the set of algebraic equations in Eqs. (42) and (43) has to be solved iteratively by using, for example, fixed-point iterations. Solving Eqs. (42) and (43) is akin to adopting an augmented Lagrangian method [see Leine and Nijmeijer (2004) and Leine (2008) for a detailed explanation]. By formulating the impact law into a simple projective iterative equation, the initial constrained problem has been transformed into an unconstrained optimization problem.

Once the solution $\dot{\mathbf{u}}_{k+1}$ and $\boldsymbol{\lambda}_{k+1}$ of Eqs. (42) and (43) has converged, the displacement $\mathbf{u}_{k+1}$ is updated according to

$$
\mathbf{u}_{k+1}=\mathbf{u}_{k}+\frac{1}{2}\left(\dot{\mathbf{u}}_{k}+\dot{\mathbf{u}}_{k+1}\right) \Delta \tau
$$

The local truncation error for displacements $\mathbf{u}$ and velocity $\dot{\mathbf{u}}$ are $\mathcal{O}\left(\Delta \tau^{3}\right)$ and $\mathcal{O}\left(\Delta \tau^{2}\right)$, respectively, for smooth motion and $\mathcal{O}(\Delta \tau)$ and $\mathcal{O}(1)$, respectively, when impulsive motion is included (Studer 2009). The pseudocode of the Moreau midpoint time-stepping scheme is shown in Algorithm 1. The complete algorithm is called HeMo. The MATLAB code is available for download (Liakou et al. 2016).

Algorithm 1. Pseudocode of Moreau's Midpoint Time-Stepping Scheme

1. The collision points are checked at midpoint displacements $\mathbf{u}_{k+1 / 2}=\mathbf{u}_{k}+\frac{1}{2} \dot{\mathbf{u}}_{k} \Delta \tau$

If contact set $\underline{\mathcal{I}}_{k+1 / 2}^{2}=\left\{i \mid \underline{\mathbf{g}}_{k+1 / 2} \leq \varepsilon\right\}$ is nonempty, move to 3; otherwise, go to

2. Smooth motion:

$$
\dot{\mathbf{u}}_{k+1}-\dot{\mathbf{u}}_{k}=-\mathbf{M}^{-1}\left(\mathbf{K} \mathbf{u}_{k+1 / 2}-\mathbf{q}_{k+1 / 2}\right) \Delta \tau
$$

3. Impact motion:

$$
\begin{aligned}
& \dot{\mathbf{u}}_{k+1}-\dot{\mathbf{u}}_{k}=\mathbf{M}^{-1}\left[-\left(\mathbf{K} \mathbf{u}_{k+1 / 2}-\mathbf{q}_{k+1 / 2}\right) \Delta \tau+\boldsymbol{\lambda}_{k+1}\right] \\
& \lambda_{k+1}^{n+1}=\operatorname{prox}_{\mathbb{R}^{+}}\left[\lambda_{k+1}^{n}-r \underline{\boldsymbol{\gamma}}_{k+1}\right]
\end{aligned}
$$$$
\text { where } n=\text { index of iterations }
$$

The iteration procedure continues until convergence, $\| \lambda_{k+1}^{n+1}-$ $\lambda_{k+1}^{n} \| \leq \varepsilon$

4. Update displacements: $\mathbf{u}_{k+1}=\mathbf{u}_{k}+\frac{1}{2}\left(\dot{\mathbf{u}}_{k}+\dot{\mathbf{u}}_{k+1}\right) \Delta \tau$

5. Next step

Moreau-Jean's $\theta$-scheme, a variation of the Moreau timestepping scheme, is presented in Appendix II, (Moreau and Panagiotopoulos 1988; Jean 1999). The two schemes have been applied to the subsequent numerical examples, but only results for the Moreau's scheme are illustrated in "Numerical Results" section, as it is found that this scheme performs faster although less controllable in terms of energy dissipation.

The critical time step for the stability of the numerical scheme depends on the phase velocity, which itself is a function of the wavelength in beam models. For a Rayleigh beam model, which is governed by Eq. (4), the phase velocity is given by (Graff 1975)

$$
c=\frac{2 \pi}{\sqrt{4 \pi^{2} \beta^{2}+\lambda^{2}}}
$$

Compared with the Euler-Bernoulli beam, where highfrequency waves travel at unbounded phase velocity, the Rayleigh phase velocity saturates to $\bar{c}=\beta^{-1}$. This makes it possible to introduce a Courant-Friedrichs-Lewy (CFL) number defined as $C=\bar{c} \Delta t / \Delta x$. For the validation problem of a cantilever Rayleigh beam (discussed in "Cantilever Beam Subjected to Impulsive Force" section), $\bar{c}=10 \quad(\beta=0.1)$. Furthermore, $\Delta \tau_{\mathrm{cr}} / \Delta x=$ 0.0577 according to an eigenvalue analysis of the discrete beam 


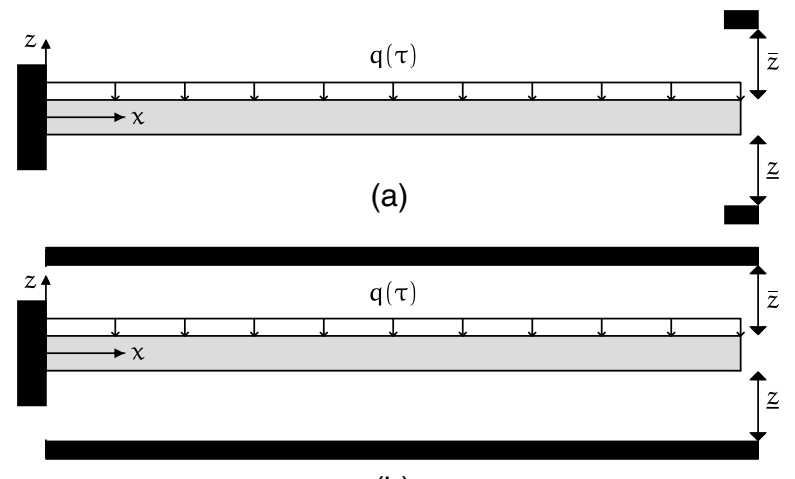

(b)

Fig. 4. Vibrating cantilever beam between two rigid obstacles: (a) discrete; (b) continuous

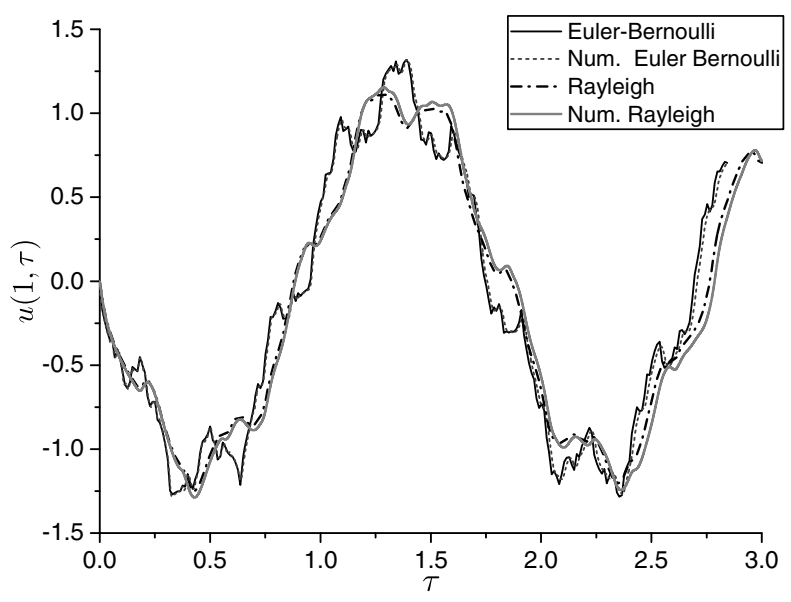

Fig. 5. Comparison between numerical and analytical solutions of end displacement $u(1, \tau)$ for a cantilever beam under impulse loading

model, $\mathbf{K u}=\omega^{2} \mathbf{M u}$, conducted for various values of $n \geq 20$, thus leading to a Courant number $C \simeq 0.577$.

\section{Numerical Results}

The computational algorithm is validated by comparing the numerical simulations with the analytical solutions of cantilever beam loaded by an impulsive transverse force at its free end. Then, the problem of a cantilever beam subjected to a harmonically time-varying, uniformly space-distributed transverse load that is vibrating between two rigid obstacles symmetrically located across the beam is analyzed. Two cases are considered: discrete stops across the free end of the beam, and two longitudinal walls (Fig. 4).

\section{Cantilever Beam Subjected to Impulsive Force}

The dynamic response of a cantilever beam subjected to an impulsive force $q(x, \tau)=\delta(x-1) \delta(\tau)$ is simulated numerically for both an Euler-Bernoulli and a Rayleigh beam with $\beta=0.1$. A first set of calculations was conducted by using $n=500$ and $\Delta \tau / \Delta x=0.01$. The results are illustrated in Fig. 5, which shows the history of the displacement at the free end, $u(1, \tau)$, calculated for $\beta=0$ and $\beta=0.1$. Also shown in Fig. 5 is the analytical solution based on a Fourier series expansion, with only the first six modes used

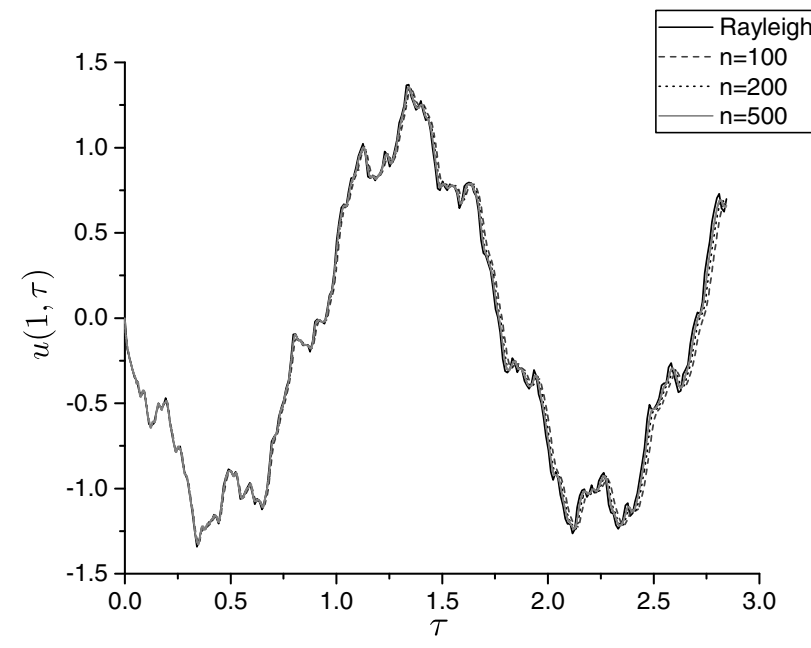

Fig. 6. Cantilever Rayleigh beam under impulse loading; influence of spatial discretization on the history of the end displacement $u(1, \tau)$

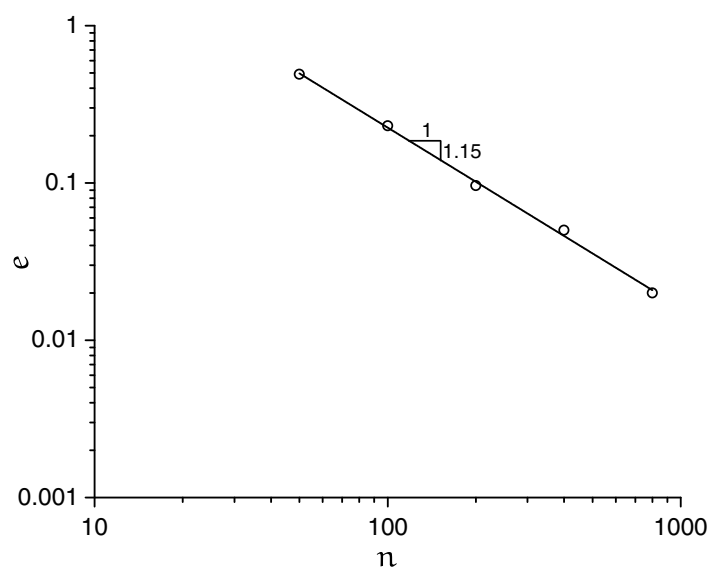

Fig. 7. Cantilever Rayleigh beam under impulse loading; influence of spatial discretization on the $l^{2}$-norm of the error on the displacement field

to represent the solution. As described in Appendix I, deriving the solution involves formulating the characteristic equation for the imposed boundary conditions, whose roots are the natural frequencies for the different modes. It can be observed that the numerical solutions faithfully reproduce the analytical ones in both cases.

The influence of the spatial discretization can be assessed from Fig. 6, which shows the evolution of $u(1, \tau)$ over $\tau \in[0,0.3]$ computed for $n=100,200$, and 500 and $\Delta \tau / \Delta x=0.01$, together with the analytical solution, for a Rayleigh beam characterized by $\beta=0.025$. In this figure, $\beta$ is small enough that the overall dynamic response of the Rayleigh beam is actually very close to that of an Euler-Bernoulli beam, as can be seen by comparing Figs. 5 and 6 . There are few visual differences between the curves, suggesting that the overall response is already captured well with $n=100$.

The discretization error is illustrated more rigorously in Fig. 7, in which the $l^{2}$-norm of the global numerical error $\left\|e_{i, k}\right\|$ is plotted versus $n ;\left\|e_{i, k}\right\|$ decreases approximately as $n^{-1}$. The error $\left\|e_{i, k}\right\|$, evaluated over a fixed time interval $\tau$, is defined as

$$
\left\|e_{i, k}\right\|=\left[\Delta \tau \Delta x \sum_{k=1}^{m} \sum_{i=i}^{n+1} e_{i, k}^{2}\right]^{1 / 2}
$$




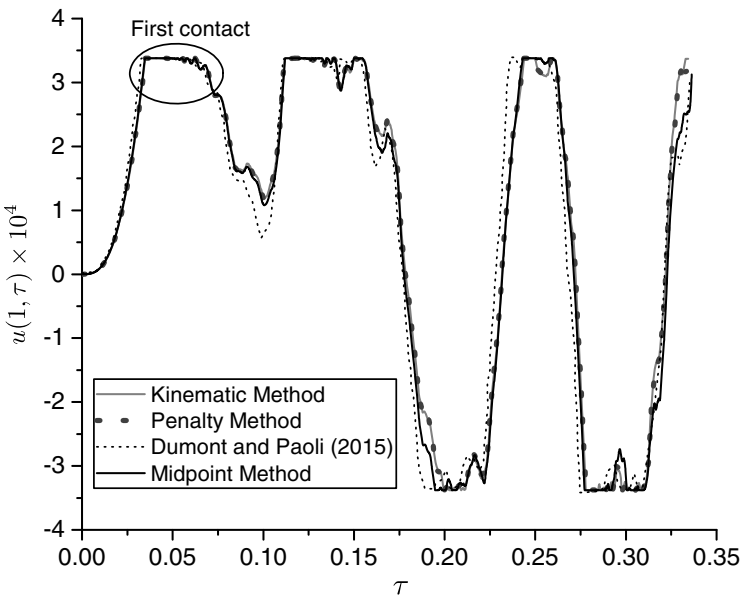

Fig. 8. History of displacement of end point of a cantilever beam vibrating between two discrete obstacles calculated with $A B A Q U S$ (kinematic and penalty method), by Dumont and Paoli (2015), and with the proposed algorithm HeMo

where $e_{i, k}=$ difference between the analytical and numerical solutions at $x=x_{i}$ and $\tau=\tau_{k}$; and $m=\tau / \Delta \tau=$ number of time steps. The computational time required to calculate the responses shown in Fig. 6 is approximately $1 \mathrm{~s}$ for $n=100$ and approximately $10 \mathrm{~s}$ for $n=500$ on a computer equipped with an Intel Core i7-3630QM CPU (Santa Clara, California) clocking at $2.4 \mathrm{GHz}$ with $8.00 \mathrm{~GB}$ of RAM.

\section{Vibrations of Euler-Bernoulli Beam Constrained by Two End Stops}

Consider now the problem of a cantilever Euler-Bernoulli beam fixed at $x=0$ and subjected to a time-harmonic uniformly distributed load $q(x, \tau)=\sin \omega \tau$ for $\tau>0$, with the beam being initially at rest, i.e., $u(x, 0)=\dot{u}(x, 0)=0, x \in[0,1]$. The beam deflections are constrained by two punctual rigid obstacles located at $\underline{z}(1)$ and $\bar{z}(1)$. This problem has been solved with a finite-element approach by Dumont and Paoli (2015), who combined some classical space discretizations (i.e., Hermite or B-spline polynomials) with velocity time-stepping schemes or a penalty method. Despite their somewhat unrealistic magnitude, the same parameter values are adopted for this study to enable comparison of the results; hence, in dimensionless form, $\omega=59.6$, and $\bar{z}(1)=-z(1)=3.37 \times 10^{-4}$ [the gap distance had to be increased by a factor 10 to match Dumont and Paoli (2015) results]. The simulations were carried out with HeMo described in this paper and with $A B A Q U S$ using the penalty and kinematic methods.

In $A B A Q U S$, the beam is modeled as a two-dimensional (2D) planar deformable beam element with a cylindrical section, while the punctual obstacles are constructed by using 2D planar analytical rigid elements. A "hard contact" was adopted for the interaction normal to the contact surface, and a "frictionless contact" for the tangential interaction. The dynamic response of the beam is computed with $A B A Q U S /$ Explicit. The default time step is assigned because it is consistent with the Courant number. For the penalty method, at least 200 elements are needed to capture the results, whereas the kinematic method requires less elements. To generate the results shown in Figs. 8 and 9, 400 elements were used for the computations with both methods.

The history of the displacement of the free end of the vibrating beam is shown in Fig. 8. The predictions made by using HeMo with
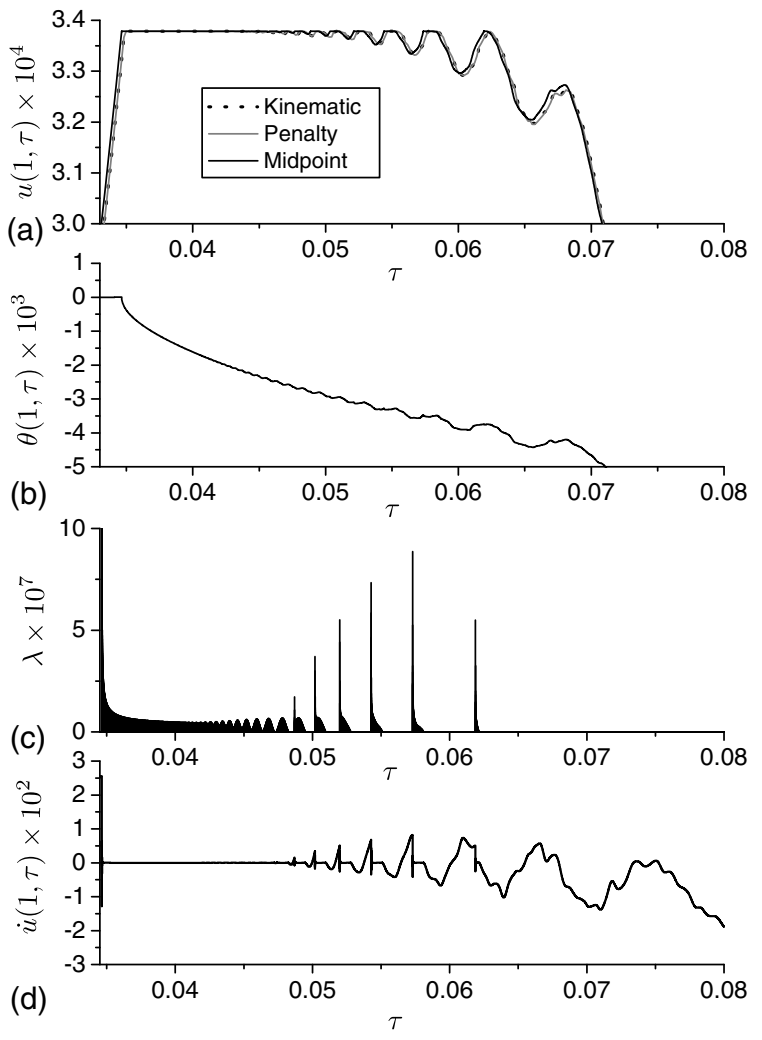

Fig. 9. Detailed response at the free end of the cantilever beam vibrating between two stops as function of time during the first contact phase ( $n=400, \Delta t / \Delta x=0.001$ ): (a) displacement $u(1, \tau)$ computed with HeMo and ABAQUS (kinematic and penalty methods); (b) inclination $\theta(1, \tau)$; (c) impulse $\lambda(1, \tau)$, noting that the impulse induced at the first impact, $\lambda \simeq 2 \times 10^{8}$, has been truncated; (d) velocity $\dot{u}(1, \tau)$

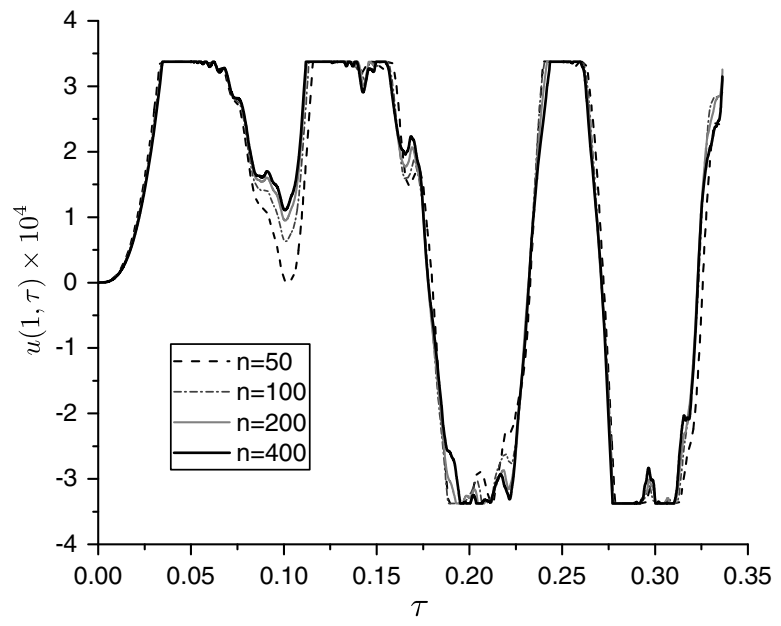

Fig. 10. History of displacement of end point of a cantilever beam vibrating between two discrete obstacles; influence of number of elements $n(\Delta t / \Delta x=0.001)$

$n=400, \Delta \tau / \Delta x=0.001$, and $e=0.5$ are in good agreement with those obtained with the kinematic and penalty methods of ABAQUS. However, the Dumont and Paoli (2015) results show larger discrepancies compared with the other predictions, as can be observed in Fig. 8. The coefficient of restitution has hardly any effect on the dynamic response of the beam in this particular example. 
Table 1. CPU Time for HeMo Computations of the Dynamical Response of a Cantilever Beam Constrained by Two Discrete Stops

\begin{tabular}{lcccr}
\hline$\Delta \tau / \Delta x$ & $n=50$ & $n=100$ & $n=200$ & $n=400$ \\
\hline 0.01 & 1.52 & 1.89 & 2.48 & 14.91 \\
0.001 & 2.87 & 7.46 & 14.89 & 123.51 \\
\hline
\end{tabular}

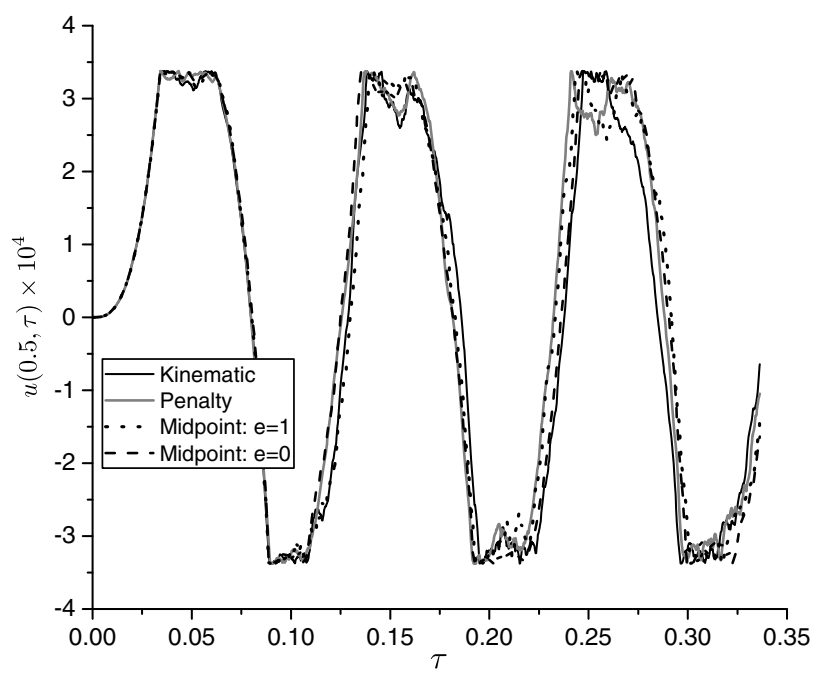

Fig. 11. History of midspan displacement of a cantilever beam constrained by two walls, obtained with different methods

Some insights about the deflection of the beam and the working of the algorithm can be gained by analyzing the results shown in Fig. 9, which illustrate the history of displacement $u$, beam inclination $\theta=u^{\prime}$, velocity $\dot{u}$, and impulse $\lambda$ at the free end of the beam during the time interval $(0.033,0.08)$ that includes the first contact phase (the first impact takes place at $\tau_{c} \simeq 0.0346$ ). The plots of $u(1, \tau)$ and $\dot{u}(1, \tau)$ in Figs. 9(a and d), respectively, indicate that the free end remains in contact with the punctual stop located at $\bar{z}(1)$ for a finite time interval lasting approximately 0.01 after the first impact at time $\tau_{c}$. During that time, the beam end rotates around the stop, as illustrated by the evolution of inclination $\theta(1, \tau)$ in Fig. 9(b). An examination of the evolution of the impulse $\lambda(1, \tau)$ during the phase of permanent contact [Fig. 9(c)] suggests that $\lambda \sim\left(\tau-\tau_{c}\right)^{-1 / 2}$.

The excellent match between the displacement $u(1, \tau)$ computed with ABAQUS (kinematic and penalty methods) and HeMo can be seen in Fig. 9(a). Furthermore, despite being a Level 2 algorithm that is designed to deal with impact by operating on impulse and velocity, the method handles well a prolonged contact phase, as confirmed by the results summarized in Fig. 9.

Fig. 10 shows that the influence of the spatial and time discretization as $n$ is varied while keeping the ratio $\Delta \tau / \Delta x=0.001$ constant. Although the overall response is captured well with $n=50$, reproducing the finer details of the displacement history of the free end requires at least $n=200$. The CPU time for various combinations of $n$ and $\Delta \tau / \Delta x$ is summarized in Table 1. Moreau's midpoint scheme is faster than the method described by Dumont and Paoli (2015), but it is slower than the penalty method.

\section{Vibrations of a Euler-Bernoulli Beam Constrained by Two Walls}

Finally, consider a problem similar to the previous one, but now with two walls equally distant from the beam in its undeformed configuration constraining its motion, i.e., $\bar{z}(i)=-\underline{z}(i)=3.37 \times 10^{-4}$ for $i \in[1, n+1]$ [Fig. 4(b)].

The history of the displacement at the midspan of the beam, $u(0.5, \tau)$, computed with HeMo $(n=400$ and $\Delta t / \Delta x=0.001)$ and with $A B A Q U S$ (using also 400 elements) is shown in Fig. 11.

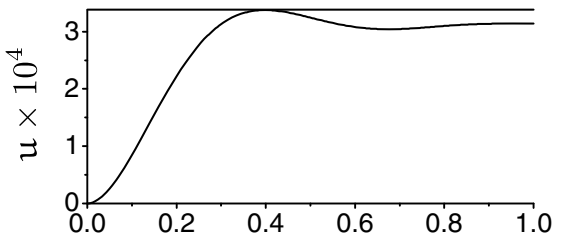

(a)

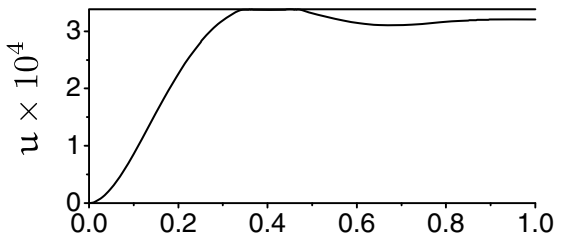

(b)

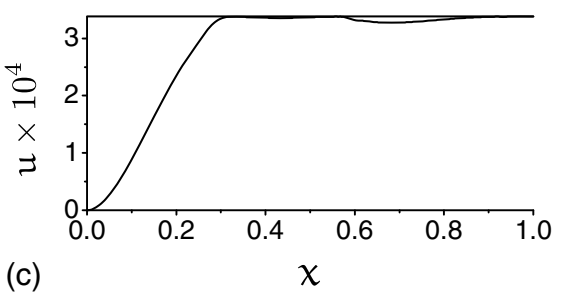

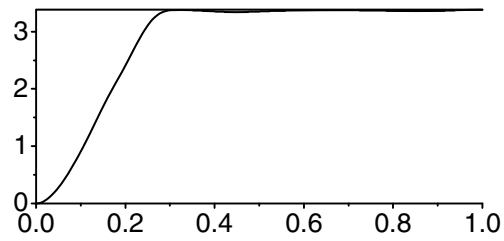

(d)

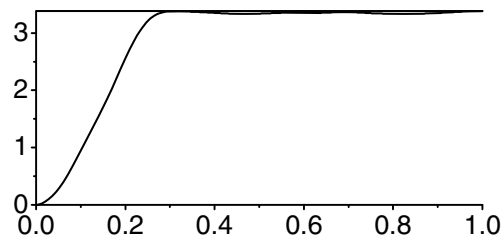

(e)

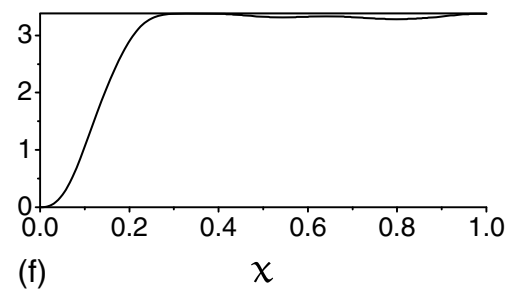

Fig. 12. Deflection profiles of the cantilever beam, $u(x, \tau)$, at various times: (a) $\tau=0.0337$ and $m=10$; (b) $\tau=0.0340$ and $m=25$; (c) $\tau=0.0347$ and $m=60$; (d) $\tau=0.0353$ and $m=28$; (e) $\tau=0.0360$ and $m=20$; (f) $\tau=0.0380$ and $m=18(n=400 ; \Delta \tau / \Delta x=0.001 ; e=0.5$; and $m=$ number of contacts) 
Table 2. CPU Time for HeMo Calculations of the Cantilever Beam Constrained by Two Walls

\begin{tabular}{lrccr}
\hline$\Delta \tau / \Delta x$ & $n=50$ & $n=100$ & $n=200$ & $n=400$ \\
\hline 0.01 & 7.91 & 21.07 & 36.24 & 65.76 \\
0.001 & 20.13 & 50.09 & 58.99 & 217.41 \\
\hline
\end{tabular}

In contrast to the previous problem, in this case, the coefficient of restitution $e$ has a nonnegligible influence on the dynamic response of the beam. For $e=1$, the response computed with HeMo compares well with that predicted by $A B A Q U S$ using the penalty method; however, for $e=0$, the response is closer to that computed by $A B A Q U S$ with the kinematic method. Nonetheless, the global response remains similar irrespective of $e$.

Fig. 12 illustrates the deflection of the beam at a few times closely after the first impact; the caption of the figure gives the number of nodes in contact at each time. The contact first takes place at approximately $x=0.4$, with the contact region rapidly spreading toward the free end of the beam over a time interval of order $O\left(10^{-4}\right)$. The contact is not continuous, however, as the beam can be seen in Figs. 12(c-f) to be detached from the wall at various places. These results confirm the robustness of the algorithm in its ability to handle multiple contacts/impacts simultaneously.

The CPU time for the HeMo calculations is provided in Table 2 for various combinations of $n$ and $\Delta \tau / \Delta x$.

\section{Conclusions}

This paper has described a numerical method to simulate the collision of a beam with rigid obstacles. The method is based on three building blocks: (1) a low-order discretization scheme that maximizes the number of nodes of the discrete beam, where impacts are detected, at the expense of the degree of continuity of the constructed displacement field; (2) the transformation of a unilateral constrained problem into an unconstrained one by using the proximal point formulation of the Signorini-type law governing the impact; and (3) the implementation of Moreau's midpoint timestepping scheme that decomposes the motion into smooth and nonsmooth components. The algorithm was validated first on the nonimpact problem of a cantilever Rayleigh beam subjected to an impulsive discrete load. Then the problem of a cantilever beam vibrating between two symmetrically located stops was analyzed. Both cases of discrete and continuous obstacles were considered, and the numerical predictions compared with published results or those obtained with a commercial code. The examples confirm the computational speed and robustness of the proposed technique and also show that multiple quasi-simultaneous impacts and prolonged contact can be handled efficiently by the algorithm.

\section{Appendix I. Free and Forced Vibration of Cantilever Rayleigh Beam}

The equations governing the free vibrations of a cantilever Rayleigh beam consist of the homogeneous equation of motion

$$
\frac{\partial^{4} u}{\partial x^{4}}+\frac{\partial^{2} u}{\partial \tau^{2}}-\beta^{2} \frac{\partial^{4} u}{\partial x^{2} \partial \tau^{2}}=0
$$

and the boundary conditions

$$
u=\frac{\partial u}{\partial x}=0, \quad \text { at } \quad x=0
$$

$$
\frac{\partial^{2} u}{\partial x^{2}}=\frac{\partial^{3} u}{\partial x^{3}}-\beta^{2} \frac{\partial^{3} u}{\partial x \partial \tau^{2}}=0, \quad \text { at } \quad x=1
$$

Assuming separation of variables, substitution of $u(x, t)=$ $U(x) T(t)$ into Eq. (46) gives

$$
\frac{d^{4} U / d x^{4}}{\left(U-\beta^{2} d^{2} U / d x^{2}\right)}=-\frac{d^{2} T / d t^{2}}{T}=\omega^{2}
$$

which leads to the following two equations:

$$
\begin{gathered}
\frac{d^{2} T}{d t^{2}}+\omega^{2} T=0 \\
\frac{d^{4} U}{d x^{4}}-\omega^{2}\left(U-\beta^{2} \frac{d^{2} U}{d x^{2}}\right)=0
\end{gathered}
$$

The solution of Eq. (50) is of the form

$$
T(t)=A \sin \omega \tau+B \cos \omega \tau
$$

where $A, B=$ unknown coefficients to be determined by the initial conditions. Additionally, setting $U(x)=e^{s x}$ into Eq. (51) leads to

$$
s^{4}+\omega^{2} \beta^{2} s^{2}-\omega^{2}=0
$$

which has two families of roots

$$
\begin{aligned}
& a=\sqrt{\left(\frac{\omega^{2} \beta^{2}}{2}\right)+\sqrt{\left(\frac{\omega^{2} \beta^{2}}{2}\right)^{2}+\omega^{2}}} \\
& b=\sqrt{\left(-\frac{\omega^{2} \beta^{2}}{2}\right)+\sqrt{\left(\frac{\omega^{2} \beta^{2}}{2}\right)^{2}+\omega^{2}}}
\end{aligned}
$$

noting that

$$
\omega=\sqrt{\frac{a^{2}-b^{2}}{\beta^{2}}}
$$

Thus, the general form of the eigenfunctions can be expressed as

$U(x)=C_{1} \sin a x+C_{2} \cos a x+C_{3} \sinh b x+C_{4} \cosh b x$

Using Eq. (53) with the boundary conditions in Eqs. (47) and (48) leads to the frequency equation

$$
2 a^{2} b^{2}+\left(a^{4}+b^{4}\right) \cos a \cosh b+\left(b^{2}-a^{2}\right) a b \sin a \sinh b=0
$$

which has an infinitre spectrum of discrete roots $\omega_{i}, i=1, \infty$. The roots depend on the slenderness ratio $\beta^{2}$. As $\beta \rightarrow 0, a \rightarrow b$, the contribution of the rotational mass becomes negligible, and the Rayleigh beam degenerates to the Euler-Bernoulli beam, which is characterized by only one wave number.

Consider now two eigenfrequencies $\left(\omega_{i}\right.$ and $\left.\omega_{j}\right)$ solutions of Eq. (54) and the corresponding normal modes $\left[U_{i}(x)\right.$ and $\left.U_{j}(x)\right]$, which both satisfy Eq. (51). To derive the orthogonality relations, multiply Eq. (51) expressed in terms of $U_{i}(x)$ by $U_{j}(x)$, and integrate it over the total length of the beam. After applying an integration by parts, one obtains

$$
\int_{0}^{1} U_{j} \frac{d^{4} U_{i}}{d x^{4}} d x=\left.\left(U_{j} \frac{d^{3} U_{i}}{d x^{3}}-\frac{d U_{i}}{d x} \frac{d^{2} U_{i}}{d x^{2}}\right)\right|_{0} ^{1}+\int_{0}^{1} \frac{d^{2} U_{j}}{d x^{2}} \frac{d^{2} U_{i}}{d x^{2}} d x
$$




$$
\begin{aligned}
& \int_{0}^{1} \omega_{i}^{2} U_{j}\left(U_{i}-\beta^{2} \frac{d^{2} U_{i}}{d x^{2}}\right) d x \\
& \quad=\int_{0}^{1} \omega_{i}^{2}\left(U_{i} U_{j}+\beta^{2} \frac{d U_{i}}{d x} \frac{d U_{j}}{d x}\right) d x-\left.\omega_{i}^{2} \beta^{2} U_{j} \frac{d^{2} U_{i}}{d x^{2}}\right|_{0} ^{1}
\end{aligned}
$$

where the left and right sides of Eq. (51) are shown separately for clarity. By applying the boundary conditions, Eqs. (55) and (56) become

$$
\int_{0}^{1} \frac{d^{2} U_{j}}{d x^{2}} \frac{d^{2} U_{i}}{d x^{2}} d x=\int_{0}^{1} \omega_{i}^{2}\left(U_{i} U_{j}+\beta^{2} \frac{d U_{i}}{d x} \frac{d U_{j}}{d x}\right) d x
$$

Similarly

$$
\int_{0}^{1} \frac{d^{2} U_{j}}{d x^{2}} \frac{d^{2} U_{i}}{d x^{2}} d x=\int_{0}^{1} \omega_{j}^{2}\left(U_{i} U_{j}+\beta^{2} \frac{d U_{i}}{d x} \frac{d U_{j}}{d x}\right) d x
$$

In view of Eqs. (57) and (58), one derives the orthogonality relation for $\omega_{i} \neq \omega_{j}$

$$
\int_{0}^{1}\left(U_{i} U_{j}+\beta^{2} \frac{d U_{i}}{d x} \frac{d U_{j}}{d x}\right) d x=0
$$

By normalizing the $i$ th normal mode $U_{i}(x)$ as

$$
\int_{0}^{1}\left(U_{i}^{2}+\beta^{2} \frac{d U_{i}}{d x} \frac{d U_{i}}{d x}\right) d x=1
$$

the final form of the orthogonality relation becomes

$$
\int_{0}^{1}\left(U_{i} U_{j}+\beta^{2} \frac{d U_{i}}{d x} \frac{d U_{j}}{d x}\right) d x=\delta_{i j}
$$

where $\delta_{i j}=$ Kronecker delta.

The general solution of a freely vibrating Rayleigh beam finally can be expressed as

$$
u(x, \tau)=\sum_{i=1}^{\infty} U_{i}(x)\left(A_{i} \sin \omega_{i} \tau+B_{i} \cos \omega_{i} \tau\right)
$$

Finally, consider a cantilever Rayleigh beam subjected to an impulsive force $(\lambda=1)$ at its free end. The displacement field, the solution of

$$
\frac{\partial^{4} u}{\partial x^{4}}+\frac{\partial^{2} u}{\partial \tau^{2}}-\beta^{2} \frac{\partial^{4} u}{\partial x^{2} \partial \tau^{2}}=\delta(\tau) \delta(x-1)
$$

is expressed as a linear combination of the normal modes, i.e.

$$
u(x, \tau)=\sum_{i=1}^{\infty} U_{i}(x) T_{i}(\tau)
$$

Substituting Eq. (64) into Eq. (63), which then is multiplied by $U_{i}$ and integrated over $[0,1]$ leads to

$$
\frac{d^{2} T_{i}}{d \tau^{2}}+\omega^{2} T_{i}=U_{i}(1) \delta(\tau)
$$

after using Eq. (51) in combination with the orthonormality condition in Eq. (61). Assuming that the beam is initially at rest, the solution of Eq. (65) is

$$
T_{i}(\tau)=\frac{U_{i}(1)}{\omega_{i}} \sin \omega_{i} \tau
$$

which provides the complete solution after substituting Eq. (66) into Eq. (64). More details can be found in Rao (2007).

\section{Appendix II. Moreau-Jean $\theta$-Method}

The Moreau-Jean $\theta$-method is an Index 2 implicit time-stepping scheme. The local error estimate is the same as Moreau's for the impulsive motion. This appendix only summarizes the main results obtained with this method [see Acary (2010) for details]. With this scheme, the equation of motion and the time discretization scheme become

$$
\begin{gathered}
\dot{\mathbf{u}}_{k+1}-\dot{\mathbf{u}}_{k}=\mathbf{M}^{-1}\left[-\left(\mathbf{K} \mathbf{u}_{k+\theta}-\mathbf{q}_{k+\theta}\right) \Delta \tau+\boldsymbol{\lambda}_{k+1}\right] \\
\mathbf{u}_{k+1}-\mathbf{u}_{k}=\Delta \tau\left[\theta \dot{\mathbf{u}}_{k+1}+(1-\theta) \dot{\mathbf{u}}_{k}\right]
\end{gathered}
$$

where the internal and external forces are expressed at a certain time along the time step by using the same interpolation,

$$
\begin{gathered}
\mathbf{K} \mathbf{u}_{k+\theta}=\theta \mathbf{K} \mathbf{u}_{k+1}+(1-\theta) \mathbf{K} \mathbf{u}_{k} \\
\mathbf{q}_{k+\theta}=\theta \mathbf{q}_{k+1}+(1-\theta) \mathbf{q}_{k}
\end{gathered}
$$

which leads to

$$
\dot{\mathbf{u}}_{k+1}-\dot{\mathbf{u}}_{k}=\hat{\mathbf{M}}^{-1}\left\{-\left[\mathbf{K}\left(\mathbf{u}_{k}+\theta \Delta \tau \dot{\mathbf{u}}_{k}\right)-\mathbf{q}_{k+\theta}\right] \Delta \tau+\lambda_{k+1}\right\}
$$

and $\hat{\mathbf{M}}$ is the iteration matrix

$$
\hat{\mathbf{M}}=\mathbf{M}+\theta^{2} \Delta \tau^{2} \mathbf{K}
$$

The contact detection also is performed at the midpoint time. The steps of the Moreau-Jean $\theta$-method are summarized in Algorithm 2.

\section{Algorithm 2. Moreau-Jean's $\theta$-Scheme}

1. The collision points are checked at midpoint time

$\mathbf{u}_{k+1 / 2}=\mathbf{u}_{k}+\dot{\mathbf{u}}_{k} \frac{\Delta \tau}{2}$

If contact set $\mathcal{I}_{k+1 / 2}=\left\{i \mid \underline{\mathbf{g}}_{k+1 / 2} \leq \varepsilon\right\}$ is nonempty, move to 3; otherwise, go to 2 .

2. Smooth motion:

$$
\dot{\mathbf{u}}_{k+1}-\dot{\mathbf{u}}_{k}=-\hat{\mathbf{M}}^{-1}\left\{\left[\mathbf{K}\left(\mathbf{u}_{k}+\theta \Delta \tau \dot{\mathbf{u}}_{k}\right)-\mathbf{q}_{k+\theta}\right] \Delta \tau\right\}
$$

3. Impact motion:

$$
\begin{aligned}
& \dot{\mathbf{u}}_{k+1}-\dot{\mathbf{u}}_{k}=\hat{\mathbf{M}}^{-1}\left\{-\left[\mathbf{K}\left(\mathbf{u}_{k}+\theta \Delta \tau \dot{\mathbf{u}}_{k}\right)-\mathbf{q}_{k+\theta}\right] \Delta \tau+\boldsymbol{\lambda}_{k+1} s\right\} \\
& \lambda_{k+1}^{n+1}=\operatorname{prox}_{\mathbb{R}^{+}}\left(\boldsymbol{\lambda}_{k+1}^{n}-r_{\gamma k+1}\right) \\
& \text { where } \hat{\mathbf{M}}=\mathbf{M}+\theta^{2} \Delta \tau^{2} \mathbf{K} \text { : the iteration matrix, }
\end{aligned}
$$

$n$ : number of iterations

The iteration procedure proceeds until convergence, $\| \lambda_{k+1}^{n+1}-$ $\lambda_{k+1}^{n} \| \leq \varepsilon$

4. Update displacements: $\mathbf{u}_{k+1}=\mathbf{u}_{k}+\Delta \tau\left[\theta \dot{\mathbf{u}}_{k+1}+(1-\theta) \dot{\mathbf{u}}_{k}\right]$

5. Next step

This method has been implemented and applied to analyze the cantilever beam impacting the two stops. The dynamic response of the beam is virtually identical to the response obtained with Moreau's scheme. Again, the choice of parameter $\theta$ and coefficient of restitution $e$ do not play an important role in the final result for this particular problem. For the problem of the cantilever beam constrained by two continuous walls, the Moreau-Jean $\theta$-method also compares well with the kinematic (for $\theta=1$ and $e=0$ ) and penalty (for $\theta=1$ and $e=1$ ) methods of $A B A Q U S$. In terms of computational time, the Moreau-Jean $\theta$-method is slower than the Moreau scheme but is still faster than Dumont and Paoli 
Table 3. Comparison of CPU Time for Punctual Obstacle between Midpoint and $\theta$-Method $(\theta=1)$ for $\Delta t / \Delta x=0.01$

\begin{tabular}{lcccc}
\hline Schemes & $n=50$ & $n=100$ & $n=200$ & $n=400$ \\
\hline Midpoint & 1.52 & 1.89 & 2.48 & 14.91 \\
$\theta$-method & 1.38 & 2.61 & 7.90 & 52.19 \\
\hline
\end{tabular}

Table 4. Comparison of CPU Time for Continuous Obstacle between Midpoint and $\theta$-Method $(\theta=1)$ for $\Delta t / \Delta x=0.01$

\begin{tabular}{lcccr}
\hline Schemes & $n=50$ & $n=100$ & $n=200$ & $n=400$ \\
\hline Midpoint & 7.91 & 21.07 & 36.24 & 65.76 \\
$\theta$-method & 5.52 & 29.97 & 94.79 & 269.56 \\
\hline
\end{tabular}

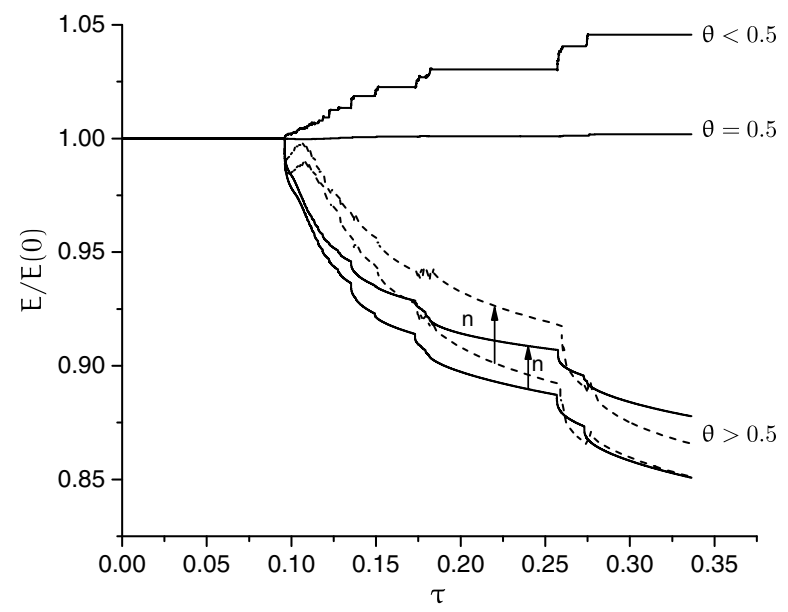

Fig. 13. Time evolution of the total mechanical energy; dashed line indicates Moreau's midpoint method, and solid line indicates Moreau-Jean's $\theta$-method

(2015). A comparison between the two schemes in terms of CPU time is presented in Tables 3 and 4 for constant ratio $\Delta t / \Delta x=0.01$ and for a representative value of the coefficient of restitution, $e=0.5$.

Besides the speed of the algorithm to complete the simulation, energy considerations also could influence the final choice of a time integrator. It is commonly recognized that energy conservation is a desired feature of a time integrator; in some instances, the possibility of adding a slight controllable amount of numerical dissipation is also appealing to ensure stability of the scheme in more complex nonlinear configurations.

For a rigid body motion, the Newton impact law for $e=1$ theoretically ensures that the energy remains constant for free vibration if there is no damping. This is not the case when the Newton impact law is applied to deformable bodies. The violation of the contact constraint at a specific point affects the velocity and, accordingly, the position of the adjacent points through the stiffness and mass matrices, $\mathbf{K}$ and $\mathbf{M}$, as they are not diagonal matrices.

Fig. 13 shows the time evolution of the total mechanical energy of the beam, defined as

$$
E=\frac{1}{2} \dot{\mathbf{u}}^{T} \mathbf{M} \dot{\mathbf{u}}+\frac{1}{2} \mathbf{u}^{T} \mathbf{K} \mathbf{u}
$$

as calculated by using the Moreau and Moreau-Jean $\theta$-schemes, for the problem of the cantilever beam vibrating between two stops. Calculations were carried out with $\Delta \tau / \Delta x=0.001, e=1$, $n=200$ and 300 for $\theta>0.5$, and $n=200$ for $\theta \leq 0.5$. Energy dissipation is observed and is reduced when the number of elements increases. For the Moreau-Jean $\theta$-method, the choice of parameter $\theta$ is also important. The energy is not bounded; therefore, the scheme is unstable for $\theta<0.5$. Energy is conserved for $e=1$ when $\theta=0.5$ [see also Acary (2014)].

\section{References}

ABAQUS [Computer software]. Dassault Systèmes, Waltham, MA.

Acary, V. (2008). "Numerical methods for nonsmooth dynamical systems: Applications in mechanics and electronics." Lecture notes in applied and computational mechanics, 1st Ed., Vol. 35, Springer, New York.

Acary, V. (2010). "An excursion into nonsmooth dynamics: From mechanics, to electronics, through control." 〈https://hal.inria.fr/inria-00569427 /document) (Oct. 2010).

Acary, V. (2014). "Energy conservation and dissipation properties of time integration methods for the nonsmooth elastodynamics with contact." $Z$. Angew. Math. Mech. 96(5), 585-603.

Andrews, K. T., Shillor, M., and Wright, S. (1996). "On the dynamic vibrations of an elastic beam in frictional contact with a rigid obstacle." J. Elast., 42(1), 1-30.

Baraff, D. (1994). Fast contact force computation for nonpenetrating rigid bodies, ACM, New York, 23-34.

Belytschko, T., Liu, W. K., and Moran, B. (2000). Nonlinear finite elements for continua and structures, 1 Ed., Wiley, New York.

Bishop, S. R., Thompson, M. G., and Foale, S. (1996). "Prediction of period-1 impacts in a driven beam." Proc. R. Soc. A, 452(1954), 2579-2592.

Brogliato, B. (1996). "Nonsmooth impact mechanics: Models, dynamics, and control." Lecture notes in control and information sciences, Vol. 220, Springer, New York.

Bruls, O., Acary, V., and Cardona, A. (2014). "Simultaneous enforcement of constraints at position and velocity levels in the nonsmooth generalized-alpha method." Comput. Methods Appl. Mech. Eng., 281, 131-161.

Cardona, A., and Geradin, M. (1988). "A beam finite element non-linear theory with finite rotations." Int. J. Numer. Methods Eng., 26(11), 2403-2438.

Challamel, N., Kocsis, A., and Wang, C. (2015). "Discrete and non-local elastica." Int. J. Non-Linear Mech., 77, 128-140.

Chen, Q. Z., Acary, V., Virlez, G., and Bruls, O. (2012). "A Newmark-type integrator for flexible systems considering nonsmooth unilateral constraints." Proc., 2nd Joint Int. Conf. on Multibody System Dynamics, Peter Eberhard, Stuttgart, Germany.

Chen, Q.-Z., Acary, V., Virlez, G., and Bruls, O. (2013). "A nonsmooth generalized alpha scheme for flexible multibody systems with unilateral constraints." Int. J. Numer. Methods Eng., 96(8), 487-511.

Depouhon, A., Detournay, E., and Denoel, V. (2014). "Accuracy of onestep integration schemes for damped/forced linear structural dynamics: Accuracy of integration schemes including forcing terms and damping." Int. J. Numer. Methods Eng., 99(5), 333-353.

Dumont, Y. (2002). "Vibrations of a beam between stops: Numerical simulations and comparison of several numerical schemes." 60(1), 45-83.

Dumont, Y. (2003). "Some remarks on a vibro-impact scheme." Numer. Algorithms, 33(1-4), 227-240.

Dumont, Y., and Paoli, L. (2015). "Dynamic contact of a beam against rigid obstacles: Convergence of a velocity-based approximation and numerical results." Nonlinear Anal.: Real World Appl., 22, 520-536.

Glocker, C. (2001). Set-valued force laws, Springer, Berlin.

Goyal, S., Perkins, N. C., and Lee, C. L. (2008). "Non-linear dynamic intertwining of rods with self-contact." Int. J. Non-Linear Mech., 43(1), $65-73$.

Graff, K. (1975). Wave motion in elastic solids, Dover, Mineola, NY.

Han, S. M., Benaroya, H., and Wei, T. (1999). "Dynamics of transversely vibrating beams using four engineering theories." J. Sound Vibr., 225(5), 935-988. 
Hencky, H. (1920). "Uber die angenaeherte loesung von stabilitaetsproblemen im raum mittels der elastischen gelenkkette." Der Eisenbau, 11, 437-452 (in German).

Ibrahim, R. A. (2009). "Vibro-impact dynamics." Lecture notes in applied and computational mechanics, Vol. 43, Springer, Berlin.

Jean, M. (1999). "The non-smooth contact dynamics method." Comput. Methods Appl. Mech. Eng., 177(3-4), 235-257.

Johansson, L. (1997). "Beam motion with unilateral contact constraints and wear of contact sites." J. Pressure Vessel Technol., 119(1), 105-110.

Klapper, I. (1996). "Biological applications of the dynamics of twisted elastic rods." J. Comput. Phys., 125(2), 325-337.

Leine, R. I. (2008). "Stability and convergence of mechanical systems with unilateral constraints." Lecture notes in applied and computational mechanics, Vol. 36, Springer, Berlin.

Leine, R. I., and Nijmeijer, H. (2004). "Dynamics and bifurcations of nonsmooth mechanical systems." Lecture notes in applied and computational mechanics, Vol. 18, Springer, Berlin.

Liakou, A., Denoel, V., and Detournay, E. (2016). "HeMo: A MATLAB program to simulated the fast in-plane dynamics of a beam with unilateral constraints." Univ. of Minnesota, Minneapolis.

Love, A. E. H. (2013). A treatise on the mathematical theory of elasticity, Cambridge University Press, Cambridge, U.K.

Melcher, J., Champneys, A. R., and Wagg, D. J. (2013). "The impacting cantilever: Modal non-convergence and the importance of stiffness matching." Philos. Trans. R. Soc. London A, 371(1993), 20120434.

Moreau, J. J., and Panagiotopoulos, P. D., eds. (1988). Nonsmooth mechanics and applications, Springer, New York.

Paoli, L. (2001). "Time discretization of vibro-impact." $R$. Soc. Philos. Trans. Math. Eng. Sci., 359(1789), 2405-2428.

Pozzolini, C., and Salaun, M. (2011). "Some energy conservative schemes for vibro-impacts of a beam on rigid obstacles." ESAIM: Math. Modell. Numer. Anal., 45(6), 1163-1192.

Rao, S. S. (2007). Vibration of continuous systems, Wiley, Hoboken, NJ.
Salvadori, M. (1951). "Numerical computation of buckling loads by finite differences." Trans. ASCE, 116(1), 590-636.

Simo, J. C. (1985). "A finite strain beam formulation. The threedimensional dynamic problem. Part I." Comput. Methods Appl. Mech. Eng., 49(1), 55-70.

Simo, J. C., and Vu-Quoc, L. (1986). "A three-dimensional finite-strain rod model. Part II: Computational aspects." Comput. Methods Appl. Mech. Eng., 58(1), 79-116.

Spillmann, J., and Teschner, M. (2007). "CoRdE: Cosserat rod elements for the dynamic simulation of one-dimensional elastic objects." Proc., 2007 ACM SIGGRAPH/Eurographics Symp. on Computer Animation, SCA '07, Eurographics Association, Aire-la-Ville, Switzerland, 63-72.

Studer, C. (2009). "Numerics of unilateral contacts and friction." Lecture notes in applied and computational mechanics, Vol. 47, Springer, Berlin.

Vyasarayani, C. P. (2009). "Transient dynamics of continuous systems with impact and friction, with applications to musical instruments." Ph.D. thesis, Univ. of Waterloo, Waterloo, ON, Canada.

Vyasarayani, C. P., McPhee, J., and Birkett, S. (2009). "Modeling impacts between a continuous system and a rigid obstacle using coefficient of restitution.” J. Appl. Mech., 77(2), 021008-021008.

Vyasarayani, C. P., Sandhu, S. S., and McPhee, J. (2012). "Nonsmooth modeling of vibro-impacting euler-bernoulli beam." Adv. Acoust. Vibr., 2012, 1-9.

Wagg, D. (2004). "A note on using the collocation method for modelling the dynamics of a flexible continuous beam subject to impacts." J. Sound Vibr., 276(3-5), 1128-1134.

Wagg, D., and Bishop, S. (2002). "Application of non-smooth modelling techniques to the dynamics of a flexible impacting beam." J. Sound Vibr., 256(5), 803-820.

Wang, C. M., Zhang, H., Gao, R. P., Duan, W. H., and Challamel, N. (2015). "Hencky bar-chain model for buckling and vibration of beams with elastic end restraints." Int. J. Struct. Stab. Dyn., 15(7), 1540007. 